\title{
Sudden death on the playing field: Can we prevent it?
}

\author{
Rohan R. Wagle ${ }^{\text {**, Melvin D. Cheitlin }}{ }^{2}$ \\ ${ }^{1}$ Baylor College of Medicine/Kelsey-Seybold Clinic (Houston, TX), USA \\ ${ }^{2}$ University of California, San Francisco (San Francisco, CA), USA
}

\begin{abstract}
From the ancient Greek Olympics to the high school gymnasium, competitive sports have become an increasingly important part of human culture. At an early age, children around the world are introduced and encouraged into sports by their parents and peers. For some, playing sports becomes a regular part of life even as they enter adulthood. The medical profession has always been encouraging of physical activity. With the rising obesity epidemic, even the recent Obama administration, spearheaded by First Lady Michelle Obama, started a campaign called "Let's Move" to promote exercise among our children. Regular physical activity has been shown to improve many markers of health and can ward off diseases fraught with high morbidity and mortality, like diabetes and obesity. However, as the intensity level of sports increases, there remains controversy as to the advantages of certain vigorous forms of exercise. In rare but dramatic circumstances, young athletes can experience sudden cardiac death on the playing field. There are some evidence-based guidelines to help the cardiologist manage the young athlete before they step foot onto the playing field, but major differences exist in different countries as to how this evidence is interpreted and how cost-effectiveness plays a role.
\end{abstract}

Accepted on July 14, 2017

\section{Epidemiology and Etiology}

Sudden cardiac death (SCD) is the leading cause of death in young athletes. SCD is defined by death occurring within one hour of onset of symptoms in someone without a previously recognized cardiovascular condition, excluding death from respiratory, cerebrovascular, traumatic, or drug-related causes [1]. The incidence of SCD is relatively low in the general population (1-2/100,000 per year), but the incidence is higher in the young athlete $(3.6 / 100,000)$ [2]. Additionally, the risk seems to be directly proportional to the competition level of the athlete. In one review, SCD incidence in high school athletes was $1 / 200,000$ per year; college athletes was $1 / 67,000$ per year; and professional athletes was $1 / 3,500$ per year [3]. Over the years, the incidence of death in young athletes has raised significantly [4]. The social impact and startling public display of the collapse of the athlete on the playing field can be very dramatic, so it calls for strategies and protocols to identify those at higher risk of adverse events.

The risk of SCD in young athletes is impacted by several variables. Males are observed to have a five times higher risk for SCD than females [5]. African-Americans carry twice the risk of SCD than Caucasians [6]. In one review of 820 sports participants in France who suffered SCD, the most common sports engaged in when SCD occurred were cycling $(31 \%)$, jogging (21\%), and soccer (13\%) [7]. In the United States, the most common sports associated with SCD are men's basketball, followed by football, together accounting for $57 \%$ of deaths [8].SCD is thought to be the result of the interplay of a variety of risk factors (Figure 1). 42 Electrical instability resulting from myocardial ischemia, left ventricular dysfunction, or channelopathies is thought to be the most common reason for sudden cardiac death. The age of the athlete can predict, with some accuracy, the most likely specific etiology of SCD. In patients less than 35 years old, SCD is most commonly due to hypertrophic cardiomyopathy $33 \%$ of deaths), less likely due to coronary anomalies involving the wrong sinus of Valsalva ( $15 \%-20 \%$ of deaths), and least likely due to arrhythmogenic right ventricular cardiomyopathy (ARVC), idiopathic dilated cardiomyopathy, and channelopathies (i.e. long QT syndrome, Brugada syndrome, and catecholaminergic VT) [9]. In patients greater than 35 years old, coronary artery pathology is the dominant etiology for SCD, most likely due to atherosclerotic disease.

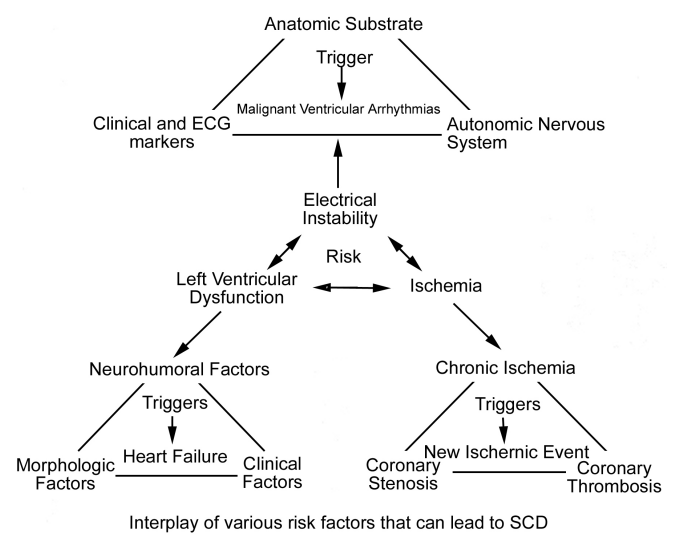

$\exists$

Figure 1. Interplay of various risk factors that can lead to SCD42 )Reproduced with permission from original author). 
In one review of 1866 deaths in young athletes in the United States, 1049 cases $(56 \%)$ were related to SCD and $690(66 \%)$ had a confirmed cardiovascular event [10]. Hypertrophic cardiomyopathy (HCM) was noted in $24 \%$ of the cases, coronary anomalies were noted in $11.3 \%$ of the cases, and possible HCM was noted in an additional $5.4 \%$ of the cases. ARVC was only noted in $2.4 \%$ of the cases, and channelopathies were diagnosed in $2.4 \%$ of cases. Premature coronary artery disease (CAD) was implicated only $2.2 \%$ of the time. Regional differences have been observed in the comparative etiologies of SCD. In comparison to Italy, the US has a higher incidence of HCM and commotio cordis in athletes experiencing SCD. However, Italy has a higher incidence of ARVC, CAD, mitral valve prolapse (MVP), and channelopathies, suggesting a possible genetic and/or environmental component in the development of these particular conditions [11]. Additionally, since Italy screens all young athletes with an electrocardiogram (ECG) and, if abnormalities are found with echocardiography, those with HCM are screened out which might account for the absence of $\mathrm{HCM}$ as a cause of sudden death in their experience [2].

\section{Pre-participation Screening}

Controversy remains in the US as to whether or not all young athletes should undergo pre-participation screening. The diseases associated with sudden cardiac death are rare. Screening athletes with physical exams and/or electrocardiograms has remained low-yield and requires large numbers of athletes to be screened to detect very few incident cases [12]. Additionally, because of physiologic cardiac adaptions in athletic individuals that can be mistaken for abnormalities, pre-participation screening can often lead to false-positive results and unnecessary anxiety and additional testing [13]. Additionally, there are not enough qualified personnel to interpret the large number of ECG's that would require accurate interpretation. Thus, the cost-effectiveness of such screening is called into question. The United States, among most countries that perform athletic pre-participation screening, recommends the use of a 14-element history for symptoms, including an in-depth family history of sudden death and cardiomyopathy, as well as a physical examination [13].

Despite the numerous debates, pre-participation screening has noticeably reduced the incidence of SCD in Italy since its implementation in the 1970's. The death rate before screening began there was $4.19 / 100,000$ person-years; this has been reduced to $0.45 / 100,000$ person-years after screening athletes with history, physicals and ECG's.2 However, this observed reduction in SCD incidence could be confounded by the greater awareness of these conditions along with excessive cautiousness by physicians and patients in taking part in competitive sports. Also, Israel has had mandatory prescreening including ECG screening and found no difference in annual mortality incidence compared to the pre-screening period of similar duration [14].

In the United States, a thorough history with an in-depth interrogation of any cardiovascular symptoms such as chest pain, syncope, pre-syncope, or palpitations is highly recommended prior to entry into competitive sports at any level.

\section{Electrocardiogram}

The electrocardiogram can be useful in detecting potential cardiac pathologies. Pre-participation ECG's are mandatory in Italy but optional in the US. Frequently, normal variants are noted to be abnormal in athletes (i.e. LVH voltage, first degree and Wenckebach A-V block, incomplete RBBB, and sinus bradycardia). Standards have been proposed to distinguish ECG findings secondary to physiologic cardiac adaptations in young athletes that do not require further evaluation from possible underlying pathology $[15,16]$ Recognizing these innocent ECG findings considerably reduces the incidence of false-positive ECG's. However, the real utility of the ECG lies in detection of certain cardiac pathologies with defined criteria. For example, HCM is associated with an abnormal ECG in over $90 \%$ of cases, including pathologic Q waves, abnormal ST-T waves and left atrial abnormality [17]. Long QT syndrome is diagnosed by the ECG, but $6 \%$ of true cases will have a QTc of 0.44 seconds or less [18]. Cases of ARVC are sometimes observed to have right ventricular conduction delays, epsilon waves, or inverted $\mathrm{T}$ waves in the initial precordial leads [19]. Nonetheless, there are notable cardiac conditions that are not appropriately screened for by ECG, including coronary anomalies, some cases of HCM, CAD, and some channelopathies [13].

In one review of 5,615 high school athletes from 30 different schools over 3 years, pre-participation screening with history, physical and ECG resulted in 582 cases (10\%) with abnormalities [20]. Cardiovascular auscultation detected 175 abnormalities (3.2\%), while ECG detected 146 abnormalities $(2.5 \%)$. When these abnormal findings were further examined including evaluation by echocardiography, 22 of the 582 cases were determined to be truly abnormal (1:255 athletes). The ECG was abnormal in 16 of the 22 true abnormal cases, and determined to have a specificity of $97.7 \%$ in detecting cardiac abnormalities potentially leading to SCD in athletes. However, the positive predictive value of an abnormal ECG detecting true cardiac pathology in young athletes is estimated at $4 \%$ [20]. In another review of 4,878 young athletes, the history/ physical had a sensitivity of $12 \%$ in predicting abnormalities that are associated with SCD while the ECG had a sensitivity of $88 \%$ [21]. However, because of the overall low incidence of SCD in young athletes, $91 \%$ of ECG abnormalities on preparticipation screening are eventually found to be falsepositives. Therefore, the 36th Bethesda Conference determined that adding an ECG to routine pre-participation screening for young athletes is not cost-effective in the US [21,22].

\section{Echocardiogram}

Using echocardiography as a mandatory screening tool is not indicated in the US. This modality is time-intensive, expensive and, because of the very low probability of finding potentially lethal abnormalities, deemed to be cost-ineffective. There are also technical limitations of the study that can lead to problems 
of under-diagnosis or over-diagnosis. For example, left ventricular hypertrophy can be physiologic (septal thickness greater than $10 \mathrm{~mm}$ and less than $12 \mathrm{~mm}$ ) or pathologic (septal thickness greater than $15 \mathrm{~mm}$ ), depending on the measurements made by the sonographer.4 Endurance athletes can sometimes have a dilated right ventricle that can be strikingly similar to what is seen in ARVC [19]. When echocardiograms were performed on 24 cyclists, $51 \%$ had LV dilatation and $12 \%$ had a left ventricular ejection fraction less than 53\% [23]. Echocardiographic criteria for left ventricular non-compaction syndrome were met in $8 \%$ of athletes in one study [24]. Coronary anomalies can often remain undiagnosed despite echocardiography, unless the coronary Ostia are defined by experienced sonographers $[25,26]$. Because of these shortcomings, as well as the potential to inappropriately exclude athletes from sport, routine echocardiography has not become standard in pre-participation screening in the US and elsewhere.

\section{Improving Outcomes}

Despite increased screening of young athletes and greater public awareness, some cases of at-risk patients will remain unrecognized and will go on to result in SCD in dramatic fashion on the playing field. Such cases are reminders of the important value of bystander assistance. Long-term and meaningful survival in patients who suffer SCD is largely determined by the time-to-defibrillation interval. Every minute that appropriate defibrillation is delayed reduces the chance for survival by $8-10 \%$. After eight minutes without defibrillation, resuscitative efforts almost always fail [43]. Having medical personnel at athletic events trained in early recognition of SCD and delivery of CPR and/or defibrillation can be a life-saving strategy for our young athletes. The 36th Bethesda conference recommended that automatic external defibrillators and personnel instructed in using the device be present at educational facilities, training centres, sports arenas and stadiums so that defibrillation can be performed in less than 5 minutes after an observed arrest [22].

\section{Specific Diseases Associated with Sudden Death in Young Athletes}

\section{Hypertrophic cardiomyopathy}

Hypertrophic Cardiomyopathy (HCM) is a genetically determined disease that affects the cellular structure of cardiac myocytes, most commonly due to mutations in one of several sarcomere genes encoding proteins that make up the contractile apparatus. HCM is characterized by left ventricular hypertrophy that can be associated with LV outflow obstruction, diastolic dysfunction, myocardial ischemia, or mitral regurgitation. In one SCD registry of 1435 young competitive athletes, HCM was thought to be the etiology in $36 \%$ of cases [25]. However, the overall prevalence of this condition is thought to be as high as 1 out of every 500 adults, according to echocardiography registries in the United States, Japan, and China [25].
Competitive athletes who are highly-trained can also develop "Athlete's heart", which is characterized by cardiac hypertrophy that can resemble HCM in many ways. HCM is usually associated with septal thickness greater than $15 \mathrm{~mm}$ and LV cavity size less than $45 \mathrm{~mm}$, while "Athlete's heart" is associated with septal thickness less than $12 \mathrm{~mm}$ and LV cavity size greater than $55 \mathrm{~mm}$ [25]. Additionally, HCM frequently has diastolic dysfunction whereas "Athlete's heart" has normal diastolic function. Left atrial enlargement and some abnormal ECG findings (sinus bradycardia, increased QRS voltage, prominent and peaked $\mathrm{T}$ waves, and $\mathrm{J}$ point elevation) can be seen in both HCM and "Athlete's heart"; however, pathologic $\mathrm{Q}$ waves, left axis deviation, and $\mathrm{T}$ wave inversions strongly favour a diagnosis of HCM [17,27]. If the diagnosis of HCM is being considered in the well-trained athlete, a period of systematic deconditioning followed by repeat echocardiography is indicated to differentiate HCM (which would exhibit no change in wall thickness) from "Athlete's heart" (wall thickness would regress greater than $2 \mathrm{~mm}$ ) [27].

The electrocardiogram is helpful as a prognostic factor in HCM. The ECG is abnormal in about $90 \%$ of patients with HCM [27]. In one study with 2485 patients with HCM, 135 patients (5\%) had a normal ECG [28]. HCM patients with a normal ECG exhibited a less severe phenotype with better cardiovascular outcomes. Cardiac MRI is helpful in detecting areas of myocardial scar, characterized by delayed gadolinium enhancement [29]. Once diagnosed, beta-blockers, nondihydropyridine calcium channel blockers, and disopyramide are recommended to reduce the left ventricular outflow obstruction and reduce angina symptoms [30]. ICD implantation is recommended in patients with a history of ventricular arrhythmias and should be considered in those with a history of unexplained syncope [31]. Patients with asymmetric septal hypertrophy and a gradient greater than $50 \mathrm{mmHg}$ across the left ventricular outflow tract should be considered for surgical myomectomy or alcohol septal ablation [32,33]. Participation in competitive sports or moderate-to-high intensity recreational activities should be restricted [41].

\section{Coronary anomalies}

Coronary anomalies come in many forms and may coexist with other forms of congenital heart disease, like Tetralogy of Fallot or transposition of the great arteries. Patients with anomalous aortic origin of a coronary artery (AAOCA) constitute more of the at-risk group because of the course that the artery might then run between the aorta and the right ventricular outflow tract, which is susceptible to sudden proximal vessel occlusion during exercise and can lead to myocardial ischemia and SCD [34]. At times, patients can present initially with symptoms of chest pain or syncope, especially during exercise [12]. However, most often, the first clinical symptom is sometimes sudden cardiac death. In one SCD registry of athletes under the age of 35 , coronary anomalies were thought to be the etiology in $13 \%$ of cases, second only to hypertrophic cardiomyopathy [35]. Diagnosis of coronary anomalies can be challenging, as the history, physical, and ECG are most often unrevealing. Skilled echo cardiographers can sometimes reveal the origin of coronary arteries from the aortic or pulmonary artery trunks 
[26]. However, coronary magnetic resonance angiography (CMRA) and coronary computed tomography angiography (CCTA) are highly sensitive and specific in identifying and characterizing these abnormalities [36]. Traditional coronary angiography can be used if other studies are not diagnostic. In symptomatic patients or those who have suffered transient $\mathrm{SCD}$, surgical correction is generally recommended if the anatomy is favourable. Until definitive correction is performed, these patients should be restricted from competitive sports as well as moderate-to-high intensity recreational activities [41].

\section{Arrhythmogenic Right Ventricular Cardiomyopathy}

Arrhythmogenic right ventricular cardiomyopathy (ARVC) is a genetically-determined disease that affects the desmosome architecture of cardiac myocytes, characterized macroscopically by a scarred appearance with fibro-fatty replacement of myocardium. This can lead to ventricular arrhythmias, right ventricular dysfunction, and sudden cardiac death. Patients can experience palpitations, syncope, or atypical chest pain at a young age (as early as 10 years old), but up to $40 \%$ of patients can remain asymptomatic upon detection [37]. When ARVC is suspected, 12-lead ECG and echocardiogram is recommended for initial diagnosis. If a confirmed diagnosis cannot be reached with these modalities, additional testing can be performed (i.e. signal-averaged ECG, $24 \mathrm{~h}$ ECG monitoring, cardiac magnetic resonance imaging, or genetic testing) [38].

Once a diagnosis of ARVC has been confirmed, it is recommended that these patients be restricted from competitive sports and high-intensity recreational activities (i.e. kayaking, rowing, boxing, triathlon). Moderate-intensity recreational activities (i.e. downhill skiing, running, surfing, martial arts, and tennis) are to be approached with caution [41]. ICD implantation is recommended in all ARVC patients with prior history of cardiac arrest or prior sustained ventricular tachycardia or ventricular fibrillation [39]. In ARVC patients with high-risk features (such as young age, prior syncope, or two or more disease-causing mutations), ICD implantation is recommended as well. Many recommend genetic testing and evaluation of family members for all patients diagnosed with ARVC [40].

\section{Summary}

Sudden death on the playing field is catastrophic because it is occurring in young people, seemingly in the best of health. Fortunately, sudden death on the playing field is rare. Nonetheless, pre-screening athletes for possible disqualifying conditions is important. Presently, a 14-component history and physical examination is universally required. The ECG can detect many asymptomatic people with potentially disqualifying conditions. However, because of the rarity of sudden death in athletes, the lack of experienced ECG interpreters, and the high incidence of false-positive and falsenegative findings, many countries (including the United States) have concluded that the pre-screening ECG is not costeffective. Other imaging techniques that are costlier are not recommended by any authority except when there is evidence of potentially disqualifying disease to establish the diagnosis. There is no recommendation prohibiting any group from including an ECG in pre-screening as long as personnel accomplished in interpreting ECGs in young athletes are involved.

It is not possible to entirely eliminate the tragic occurrence of sudden death on the playing field. However, with the use of pre-screening and adequate preparation for its rare incidence, most of the possible tragedies can be avoided.

\section{References}

1. Montagnana M, Lippi G, Franchini M, et al. Sudden cardiac death in young athletes. Intern Med. 2008;47:1373-8

2. Corrado D, Basso C, Pavei A, et al. Trends in sudden cardiovascular death in young competitive athletes after implementation of a preparticipation screening program. JAMA. 2006;296:1593-1601.

3. Drezner JA. Contemporary approaches to the identification of athletes at risk for sudden cardiac death. Curr Opin Cardiol. 2008;23:494-501.

4. Maron BJ. Distinguishing hypertrophic cardiomyopathy from athlete's heart physiological remodelling: clinical significance, diagnostic strategies and implications for preparticipation screening. $\mathrm{Br} \mathrm{J}$ Sports Med. 2009;43:649-56.

5. Van Camp SP, Bloor CM, Mueller FO, et al. Nontraumatic sports death in high school and college athletes. Med Sci Sports Exerc. 1995;27:641-7.

6. Maron BJ, Gardin JM, Flack JM, et al. Prevalence of hypertrophic cardiomyopathy in a general population of young adults. Echocardiographic analysis of 4111 subjects in the CARDIA Study. Coronary Artery Risk Development in (Young) Adults. Circulation. 1995;92:785-9.

7. Marijon E, Tafflet M, Celermajer DS, et al. Sports-related sudden death in the general population. Circulation. 2011;124:672-81.

8. Harmon KG, Asif IM, Maleszewski JJ, et al. Incidence and Etiology of Sudden Cardiac Arrest and Death in High School Athletes in the United States. Mayo Clin Proc. 2016;91:1493-1502.

9. Maron BJ, Zipes DP, Kovacs RJ. Eligibility and Disqualification Recommendations for Competitive Athletes With Cardiovascular Abnormalities: Preamble, Principles, and General Considerations : A Scientific Statement From the American Heart Association and American College of Cardiology. J Am Coll Cardiol. 2015; 66:2343-49.

10. Maron BJ, Doerer JJ, Haas TS, et al. Sudden Deaths in Young Competitive Athletes Analysis of 1866 Deaths in the United States, 1980-2006. Circulation. 2009;119:1085-92.

11. Link MS, Mark Estes NA 3rd. Sudden cardiac death in athletes. Prog Cardiovasc Dis. 2008;51:44-57. 
12. Maron BJ, Shirani J, Poliac LC, et al. Sudden Death in Young Competitive Athletes Clinical, Demographic, and Pathological Profiles. JAMA.1996;276:199-204.

13. Maron BJ, Friedman RA, Kligfield P, et al. Assessment of the 12-lead electrocardiogram as a screening test for detection of cardiovascular disease in healthy general populations of young people (12-25 years of age): a scientific statement from the American Heart Association and the American College of Cardiology. J Am Coll Cardiol. 2014; 64:1479-514.

14. Steinvil A, Chundadze T, Zeltser D, et al. Mandatory Electrocardiographic Screening of Athletes to Reduce Their Risk for Sudden Death : Proven Fact or Wishful Thinking?. J Am J Cardiol 2011;57:1291-6.

15. Corrado D, Pelliccia A, Heidbuchel H, et al. Recommendations for interpretation of 12-lead electrocardiogram in the athlete. Eur Heart J. 2010;31:243-59.

16. Brosnan M, La Gerche A, Kalman J, et al. The Seattle Criteria increase the specificity of preparticipation ECG screening among elite athletes. $\mathrm{Br} \mathrm{J}$ Sports Med. 2014;48:1144-50.

17. Drezner JA, Ashley E, Baggish AL, et al. Abnormal electrocardiographic findings in athletes: recognising changes suggestive of cardiomyopathy. Br J Sports Med. 2013;47:137-52.

18. Vincent GM, Timothy KW, Leppert M, et al. The spectrum of symptoms and QT intervals in carriers of the gene for the long-QT syndrome. N Engl J Med. 1992;327:846-52.

19. Zaidi A, Sheikh N, Jongman JK, et al. Clinical Differentiation Between Physiological Remodeling and Arrhythmogenic Right Ventricular Cardiomyopathy in Athletes With Marked Electrocardiographic Repolarization Anomalies. J Am Coll Cardiol. 2015;65:2702-11.

20. Fuller CM, McNulty CM, Spring DA, et al. Prospective screening of 5,615 high school athletes for risk of sudden cardiac death. Med Sci Sports Exerc. 1997;29:1131-8.

21. Asif IM, Drezner JA. Sudden Cardiac Death and Preparticipation Screening: The Debate Continues-In Support of Electrocardiogram-Inclusive Preparticipation Screening. Progress in Cardiovascular Diseases 2012;54:445-50.

22. Maron BJ, Zipes DP. 36th Bethesda Conference: Eligibility Recommendations for Competitive Athletes With Cardiovascular Abnormalities. J Am Coll Cardiol. 2005;45:1313-75.

23. Abergel E, Chatellier G, Hagege AA, et al. Serial left ventricular adaptations in world-class professional cyclists: implications for disease screening and follow-up. J Am Coll Cardiol. 2004;44:144-9.

24. Gati S, Chandra N, Bennett RL, et al. Increased left ventricular trabeculation in highly trained athletes: do we need more stringent criteria for the diagnosis of left ventricular non-compaction in athletes? Heart. 2013;99:401-8.
25. Maron BJ, Thompson, PD, Ackerman MJ, et al. Recommendations and Considerations Related to Preparticipation Screening for Cardiovascular Abnormalities in Competitive Athletes: 2007 Update Circulation. 2007;115:1643-55.

26. Owens DS, Prutkin JM, Salerno JC, et al. The 5-minute screening echocardiogram for athletes. J Am Soc Echocardiogr. 2008;21:786-8.

27. Lauschke J, Maisch B. Athlete's heart or hypertrophic cardiomyopathy? Clin Res Cardiol. 2009;98:80-8.

28. McLeod CJ, Ackerman MJ, Nishimura RA, et al. Outcome of patients with hypertrophic cardiomyopathy and a normal electrocardiogram. J Am Coll Cardiol. 2009;54:229-33.

29. La Gerche A, Baggish AL, Knuuti J, et al. Cardiac Imaging and Stress Testing Asymptomatic Athletes to Identify Those at Risk of Sudden Cardiac Death. J Am Coll Cardiol Img. 2013;6:993-1007.

30. Sherrid MV, Pearle G, Gunsburg DZ. Mechanism of benefit of negative inotropes in obstructive hypertrophic cardiomyopathy. Circulation. 1998;97:41-7.

31. Maron BJ, Rowin EJ, Casey SA, et al. How Hypertrophic Cardiomyopathy Became a Contemporary Treatable Genetic Disease With Low Mortality: Shaped by 50 Years of Clinical Research and Practice. JAMA Cardiol. 2016;1:98-105.

32. Robbins RC, Stinson EB. Long-term results of left ventricular myotomy and myectomy for obstructive hypertrophic cardiomyopathy. J Thorac Cardiovasc Surg. 1996;111:586-94.

33. Kim LK, Swaminathan RV, Looser P, et al. Hospital Volume Outcomes After Septal Myectomy and Alcohol Septal Ablation for Treatment of Obstructive Hypertrophic Cardiomyopathy: US Nationwide Inpatient Database, 2003-2011. JAMA Cardiol. 2016;1:324-32.

34. Cheitlin MD, MacGregor J. Congenital Anomalies of Coronary Arteries. Herz 2009;34:268-79.

35. Maron BJ, Carney KP, Lever HM, et al. Relationship of race to sudden cardiac death in competitive athletes with hypertrophic cardiomyopathy. J Am Coll Cardiol. 2003;41:974-80.

36. Prakken NH, Velthuis BK, Cramer MJ, et al. Advances in cardiac imaging: the role of magnetic resonance imaging and computed tomography in identifying athletes at risk. Br J Sports Med. 2009;43:677-84.

37. Hulot JS, Jouven X, Empana JP, et al. Natural history and risk stratification of arrhythmogenic right ventricular dysplasia/cardiomyopathy. Circulation. 2004;110:1879-84.

38. Marcus FI, McKenna WJ, Sherrill D, et al. Diagnosis of arrhythmogenic right ventricular cardiomyopathy/ dysplasia: proposed modification of the task force criteria. Circulation. 2010;121:1533-41.

39. Epstein AE, DiMarco JP, Ellenbogen KA, et al. 2012 ACCF/AHA/HRS focused update incorporated into the ACCF/AHA/HRS 2008 guidelines for device-based therapy of cardiac rhythm abnormalities: a report of the American College of Cardiology Foundation/American 
Heart Association Task Force on Practice Guidelines and the Heart Rhythm Society. J Am Coll Cardiol. 2013;61:e6-75.

40. Sen-Chowdhry S, Morgan RD, Chambers JC, et al. Arrhythmogenic cardiomyopathy: etiology, diagnosis, and treatment. Annu Rev Med. 2010;61:233-53.

41. Mitchell JH, Haskell W, Snell P, et al. Task Force 8: classification of sports. J Am Coll Cardiol. 2005;45:1364-7.

42. Sovari AA. Sudden Cardiac Death. Medscape. emedicine.medscape.com/article/151907-overview. April 28, 2014.
43. Callans DJ. Out-of-Hospital Cardiac Arrest - The Solution is Shocking. NEJM. 2004;35:632-634.

\section{*Correspondence to}

Rohan R. Wagle

Baylor College of Medicine/Kelsey-Seybold Clinic

Houston, TX

USA 